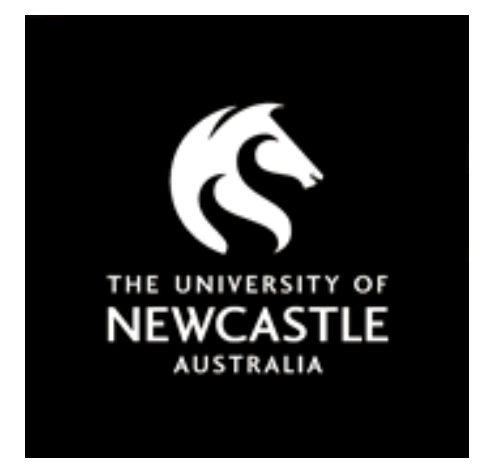

\title{
NOVA
}

University of Newcastle Research Online

nova.newcastle.edu.au

Palliser, Hannah K.; Kelleher, Meredith A.; Welsh, Toni N.; Zakar, Tamas; Hirst, Jonathon J. “ Mechanisms leading to increased risk of preterm birth in growth-restricted guinea pig pregnancies". Originally published in Reproductive Sciences Vol. 21, Issue 2, p. 269-276 (2014)

Available from: http://dx.doi.org/10.1177/1933719113497268

Accessed from: $\underline{\text { http://hdl.handle.net/1959.13/1059074 }}$ 


\section{Mechanisms leading to increased risk of preterm birth in growth restricted guinea pig}

\section{pregnancies}

Hannah K Palliser ${ }^{1,2} \mathrm{PhD}$, Meredith A Kelleher ${ }^{1,2}$, Toni Welsh ${ }^{2,3} \mathrm{PhD}$, Tamas Zakar ${ }^{2,3} \mathrm{MD}, \mathrm{PhD}$, and Jonathan J Hirst ${ }^{1,2}$ PhD.

${ }^{1}$ School of Biomedical Sciences, University of Newcastle, NSW, Australia

${ }^{2}$ Mothers and Babies Research Centre, Hunter Medical Research Institute, Newcastle, NSW, Australia

${ }^{3}$ School of Medicine and Public Health, University of Newcastle, NSW, Australia.

This work was conducted at the Mothers and Babies Research Centre, Hunter Medical Research Institute, Newcastle, NSW, Australia

This work was supported by the John Hunter Hospital Charitable Trust and a University of Newcastle Research Fellowship awarded to HKP.

Corresponding Author:

Hannah Palliser, PhD

MBRC/HMRI

Locked bag 1

Hunter Region Mail Centre

NSW 2310

Australia

$\mathrm{Ph}+61240420371$

Fax +61 249214394

Hannah.palliser@newcastle.edu.au

Meredith.kelleher@uon.edu.au

Toni.welsh@newcastle.edu.au

Tamas.zakar@newcastle.edu.au

Jon.hirst@newcastle.edu.au 


\section{ABSTRACT}

2 Intrauterine growth restriction (IUGR) is a risk factor for preterm labor however the mechanisms

3 of the relationship remain unknown. Prostaglandin (PG), key stimulants of labor, availability is

4 regulated by the synthetic enzymes prostaglandin endoperoxidase 1 and 2 (PTGS1 and 2) and the

5 metabolising enzyme 15-hydroxyprostaglandin dehydrogenase (HPGD). We hypothesised that

6 IUGR increases susceptibility to preterm labor due to the changing balance of synthetic and

7 metabolising enzymes and hence greater PG availability. We have tested this hypothesis using a

8 surgically induced IUGR model in guinea pigs, which results in significantly shorter gestation.

9 Myometrium, amnion, chorion and placentas were collected from sham-operated or IUGR

10 pregnancies and PTGS1 and HPGD protein expression were quantified throughout late gestation

11 (>62days) and labor. PTGS1 expression was significantly upregulated in the myometrium of

12 IUGR animals and chorionic HPGD expression was markedly decreased $(\mathrm{P}<0.01$ and $\mathrm{P}<0.001$,

13 respectively). These findings suggest a shift in the balance of PG production over metabolism in

14 IUGR pregnancies leads to a greater susceptibility to preterm birth.

16 Keywords: Growth restriction, Preterm labor, 15- hydroxyprostaglandin dehydrogenase, 17 Prostaglandins, Myometrium 


\section{INTRODUCTION}

20 Preterm labor remains a major problem and despite the seriousness of the consequences of

21 preterm delivery, effective treatment and prevention remain elusive. Not only is intrauterine

22 growth restriction (IUGR) a major risk factor for preterm labor but IUGR neonates born preterm

23 have greater morbidity ${ }^{1-5}$. Difficulties in the development of preventative approaches stem from

24 the paucity of knowledge over the underlying relationship between risks factors and processes of 25 preterm labor.

27 Previous studies by us and others have shown the guinea pig is an optimal small animal for use 28 in studying the induction of labor ${ }^{6-10}$. In particular this species has a relatively long gestation, 29 progesterone is produced by the placenta and does not decline until the delivery of the placenta

3011 . We have previously used a guinea pig model of IUGR to examine effects on myometrial 31 progesterone receptor isoform expression as term approaches and identified a progesterone 32 withdrawal mechanism at labor similar to that identified in women ${ }^{10}$. Furthermore, using this 33 model we showed that while IUGR did alter PR expression levels, the changes seen would be 34 unlikely to account for the increased vulnerability to preterm labor.

36 There is some commonality between changes in inflammatory processes shown to be involved in 37 the regulation of labor and some of the intrauterine changes associated with IUGR pregnancies.

38 These include the upregulation of proinflammatory cytokines known to stimulate prostaglandin

39 synthesis ${ }^{12-14}$. There is also evidence that proinflammatory processes suppress prostaglandin 40 metabolism in intrauterine tissue and therefore the availability of prostaglandins at the contractile 41 site of action, the myometrium ${ }^{15}$. These changes may make the IUGR pregnancy vulnerable to 
42 triggering processes that raise prostaglandin production in the uterine compartment and thus may

43 explain the onset of preterm labor in some IUGR pregnancies.

44

45 Prostaglandins are well recognised as regulators of myometrial contractions, membrane rupture

46 and cervical dilatation in many species ${ }^{16,17}$. The regulation of their synthesis and metabolism at

47 term spontaneous labor has been well investigated with the expression and activity of the rate

48 limiting synthetic enzyme prostaglandin $\mathrm{H}$ synthase (PTGS) found to increase and the

49 metabolising enzyme 15-hydroxyprostaglandin dehydrogenase (HPGD) to decrease in uterine

50 tissues ${ }^{18-21}$.

51

52 The effect of pregnancy complications associated with preterm labor on PTGS and HPGD is less

53 clear and we aimed to investigate the effect of IUGR on these key components of the

54 prostaglandin synthesis and metabolism pathway. The identification of the IUGR-induced

55 changes provides insight into pathways stimulated by fetal compromise and clarifies the role of

56 these pathways in the mechanism of preterm labor.

57 


\section{METHODS}

\section{Animals}

60 Outbred tri-color guinea pigs were time mated at the Research Support Unit of the University of

61 Newcastle, Australia. All animal work was carried out in accordance with the University of

62 Newcastle Animal Care and Ethics Committee. In order to establish placental insufficiency and

63 subsequent IUGR in guinea pig fetuses, a modification of the method of Turner and Trudinger

64 was used $^{22}$ as previously described ${ }^{10}$. Briefly surgery was performed at day 32-35 of gestation

65 (term approximately 71 days). The uterine horns were exposed and the uterine artery and the

66 branches (spiral arteries) feeding each placental site identified. Diathermy was used to ablate

67 half the arteries supplying each placenta. Sham surgeries in which uterine artery branches were

68 exposed but not ablated were performed in order to obtain control tissues. Dams were

69 euthanized at day 62 of gestation ( control n=6; IUGR n=6), day 65 (control n=6; IUGR n=6),

70 day 68 (control $n=9$; IUGR $n=9$ ) and during labor (control $n=6$; IUGR $n=5$ ) by $\mathrm{CO}_{2}$ inhalation.

71 Labor was identified by the birth of the first pup. After euthanasia, fetuses were removed and

72 placement in horn, sex, body, brain and liver weights were recorded. Placenta, amnion, chorion

73 and myometrial samples collected from the site of each fetal head were snap-frozen in liquid

74 nitrogen and stored at $-80^{\circ} \mathrm{C}$. No significant difference was found in litter size, fetal viability,

75 sex and placement of fetuses within the uterine horn (data not presented) between sham-operated

76 and IUGR animals. No more than one fetus and its associated uterine tissues of each sex was

77 used from each litter. 


\section{Western blotting}

80 Frozen myometrial, placental, amnion and chorion tissues were pulverized on dry ice and protein

81 extracted. Briefly, samples $(100 \mathrm{mg})$ were homogenized in $1 \mathrm{ml}$ ice cold buffer $(50 \mathrm{mM}$ Tris- $\mathrm{HCl}$

82 (pH7.5), 150mM NaCl, 1\% NP-40, 0.5\% Na Deoxycholate, 0.1\% SDS) containing Complete

83 Protease Inhibitor Cocktail and PhosphoSTOP Phosphatase Inhibitor Cocktail (Roche

84 Diagnostics, Castle Hill, Australia). After centrifugation, the supernatant was collected and

85 protein content determined using colorimetric detection and quantitation (Pierce Protein Assay

86 kit, ThermoFisher Scientific, Rockford, USA). Proteins (15 $\mathrm{g}$ amnion; 15ug myometrium; $20 \mathrm{ug}$

87 placenta) were separated using 10\% Bis-Tris polyacrylamide pre cast gels (Invitrogen, Mt

88 Waverley, Australia) and transferred to PVDF (Hybond-P, GE Healthcare, Sydney, Australia) by

89 electroblotting. Membranes were then blocked in 5\% skim milk in TBST (25mM Tris-HCl,

$9015 \mathrm{mM} \mathrm{NaCl}, 0.1 \% \mathrm{v} / \mathrm{v}$ Tween-20) at room temperature for 1 hour. Membranes were incubated

91 overnight at $4^{\circ} \mathrm{C}$ in a 1:500 dilution of goat anti PTGS1 antibody (Santa Cruz, California, USA)

92 in TBST containing 5\% skim milk. After washing ( 5 x $5 \mathrm{~min}$ in TBST), the membranes were

93 incubated in a 1:2000 dilution in 5\% skim milk in TBST of anti-goat IgG (HRP-conjugated,

94 Dako, Glostrup, Denmark) for 1 hour at room temperature. The immune complexes were

95 visualized using SuperSignal West Pico chemiluminescent substrate (Pierce, Thermo Fisher

96 Scientific) detection system and captured using the LAS-3000 Imaging System (Fuji Photo Film,

97 Tokyo, Japan). Determination of HPGD protein expression in the chorion was carried out as

98 above with the following changes: 60ug protein per lane were electrophoresed and the PVDF

99 membrane was air dried and reactivated in methanol following transfer. The primary antibody

100 (rabbit anti HPGD, Cayman Chemicals, Michigan, USA) was incubated at a dilution of 1:200, 
101 followed by washes in 5\% skim milk before incubation with anti-rabbit IgG (HRP-conjugated,

102 Upstate, Millipore, MA, USA). Pre-adsorbed antibody-peptide controls were run with each

103 tissue type to determine specificity of PTGS1 and HPGD detection (Figure 1). Relative amounts

104 of expression were quantified by optical density analysis using Multi Gauge v3.0 software (Fuji,

105 Photo Film) after stripping and reprobing for $\beta$-actin (ab8227, Abcam, Cambridge, USA). In this

106 procedure, PTGS1 and HPGD band intensities were normalized to a calibrator sample (internal

107 control) run on every blot (PTGS1, pooled myometrial sample; HPGD, pooled chorion sample)

108 and to $\beta$-actin to allow for comparison between blots and to correct for loading variance

109 respectively.

110

111 Amniotic fluid and maternal plasma cortisol concentrations

112 Free cortisol concentrations in amniotic fluid were measured using Salimetrics Salivary Cortisol

113 Enzyme Immunoassay kits as per manufacturers instructions (State College, PA, USA). Briefly,

11496 well plates coated with monoclonal antibodies against cortisol were loaded with cortisol

115 standards, controls and unknown samples run in duplicate. The plates were incubated with

116 cortisol enzyme conjugate, washed, and incubated with substrate solution. The plate was read on

117 a Fluostar Optima plate reader (BMG Labtech, Germany) at 450nm. Data was analyzed with a 4

118 parameter fit sigmoid standard curve and unknown sample concentrations calculated (Graphpad

119 Software Inc, La Jolla, CA, USA). Inter- and intra- assay coefficients were $6.89 \%$ and $5.52 \%$

120 respectively. Total cortisol concentrations in amniotic fluid and cortisol and progesterone

121 concentrations in maternal plasma were measured by the Hunter New England Area Pathology

122 using the UniCel Dx1800 Access Immunoassay systems as per manufacturers instructions

123 (Beckman Coulter Inc, Gladesville, NSW, Australia) and as briefly described above. The inter 
124 and intra assay coefficients of variance were $5.17 \%$ and $4.3 \%$ (cortisol) and $9.8 \%$ and $7.9 \%$ 125 (progesterone) respectively.

126

127 Statistical analyses

128 Data are shown as mean \pm SEM. All data were analyzed using PASW statistical software

129 (Version 18, SPSS Inc., Chicago, IL, USA). Two-way measures ANOVA were used to compare

130 control with IUGR at each gestational age. Subsequent Bonferroni post hoc tests were used to

131 assess differences between groups. Spearman correlations were used to assess the relationship

132 between HPGD expression and fetal body weight. $P<0.05$ was considered to be statistically 133 significant.

134 


\section{RESULTS}

\section{Effect of placental artery ablation}

137 The placental artery ablation used in the current study induced significant growth restriction with

138 lower body weights in the fetuses subjected to artery ablation (Table $1, \mathrm{P}<0.001$ ). These fetuses

139 also showed a marked increase in brain to liver ratio compared to fetuses where the sham

140 procedure was performed, indicating that the ablation resulted in significant asymmetric growth

141 with brain sparing. Placental weight was also significantly lower following IUGR induction

142 surgery $(\mathrm{P}<0.0001)$. Gestation length was significantly shorter in the IUGR pregnancies

143 compared to controls $(69.0 \pm 0.63, \mathrm{n}=5$ and $70.9 \pm 0.4$ days, $\mathrm{n}=6$, respectively; $\mathrm{P}<0.05)$.

145 Effect of IUGR induction on prostaglandin endoperoxide synthase expression

146 PTGS1 expression rose with advancing gestation in the placenta (Figure $2 \mathrm{~A}, \mathrm{P}<0.0001$ ) and

147 amnion (Figure $2 \mathrm{~B}, \mathrm{P}<0.01$ ) with maximal levels reached by GA68. There were no differences

148 in the level of expression between control and IUGR animals at any gestational age examined

149 (Figure 2A and B). In contrast, PTGS1 expression in the myometrium did not rise over the 62-

15068 day period but was markedly higher at 68 days of gestation in animals subjected to IUGR

151 induction surgery compared to sham operated controls (Figure $3 \mathrm{~A}, \mathrm{P}<0.01$ ). In addition, when

152 the data were recalculated from the time of expected delivery (GA71 for control and GA69 for

153 IUGR animals as calculated from laboring groups above), myometrial PTGS1 expression

154 remained markedly higher in the IUGR animals compared to controls two days before expected

155 delivery (Figure 3B, $\mathrm{P}<0.05$ ). Furthermore, myometrial PTGS1 expression in control

156 pregnancies was significantly increased at labor (compared to GA62 levels, $\mathrm{P}<0.05$ ) whilst in

157 IUGR pregnancies there was no increase at labor compared to 62-68 day expression levels. 
159 Effect of IUGR induction on prostaglandin dehydrogenase expression

160 Levels of HPGD protein expression in the chorion did not change between 62 and 68 days of 161 gestation (Figure 4A). Expression was however markedly lower in the chorion of IUGR animals 162 at 62 and 68 days compared to controls $(\mathrm{P}<0.001)$ and showed a trend toward lower expression 163 at 65days. Examining HPGD expression based on time before expected delivery (GA71 for 164 controls, GA69 for IUGR pregnancies) showed that this reduction in expression occurred at least 1657 days prior to delivery (Figure 4B, $\mathrm{P}=0.03$ ). After labor onset, chorionic HPGD expression in 166 controls dropped significantly compared to GA68 levels, however no such decrease was 167 observed in chorion from IUGR pregnancies. When HPGD expression was correlated with fetal 168 body weight at GA68 in individual fetuses, a significant positive correlation was observed 169 (Figure 5; $\mathrm{r}=0.56, \mathrm{P}=0.015$ ) showing that the smallest fetuses had the lowest expression of 170 HPGD protein in the chorion.

171

172 Circulating maternal and amniotic fluid cortisol and progesterone concentrations

173 Total cortisol concentrations in amniotic fluid rose at term in IUGR fetuses and markedly with 174 the onset of labor in control animals (Figure 6A, $\mathrm{P}<0.001$ ). Amniotic fluid cortisol 175 concentrations did not differ between IUGR and control animals except at labor where, in 176 contrast to the controls, cortisol in amniotic fluid from IUGR animals demonstrated no further 177 increase with labor onset $(\mathrm{P}<0.001)$. Circulating maternal plasma cortisol concentrations did not 178 differ between control and IUGR pregnancies in any gestational group. However, mothers in the 179 sham-operated group exhibited an increase in circulating cortisol concentrations at labor 
180 compared to levels at GA68 (Figure 6B, $\mathrm{P}=0.016$ ) whilst no change was seen in mothers with 181 IUGR foetuses over late gestation or labor.

182 Circulating maternal progesterone concentrations ranged from 300-600 $\mathrm{ng} / \mathrm{ml}$ however did not 183 differ between control and IUGR pregnancies nor between gestational ages (data not presented). 184 


\section{DISCUSSION}

186 Consistent with the present findings, we have previously shown that the surgical intervention

187 used in the current study causes a reduction in fetal growth with a marked increase in brain to

188 liver ratio ${ }^{10,23}$. These changes are consistent with a limitation of placental function such that

189 nutrient delivery is limited and growth is reduced with significant brain (head) sparing and

190 asymmetric growth as seen with IUGR in human pregnancy ${ }^{24}$. The key findings of the study

191 were that this disruption to growth is associated with an increase in prostaglandin synthetic

192 capacity and a concurrent reduction in the potential of the chorion to protect the myometrium

193 from elevated prostaglandin exposure due to lower HPGD levels.

195 There is extensive evidence showing that pregnancies complicated by IUGR have a high

196 incidence of preterm labor ${ }^{2,3}$. Although not all IUGR pregnancies deliver before term, this

197 compromise is a key risk factor and suggests that factors limiting fetal growth also increase

198 susceptibility for preterm birth. The present finding suggests this vulnerability stems from an

199 increase in the availability of key stimulatory prostaglandins at the myometrium and this may

200 increase to a point where normal endocrine changes that occur with advancing gestation may

201 become sufficient to trigger the onset of labor or make these pregnancies more vulnerable to

202 secondary insults and associated stimulation. While the difference in delivery time is relatively

203 modest, potentially representing a week if directly translated to the human gestation length, the

204 changes in expression of enzymes regulating prostaglandin availability occur up to 7 days before

205 term suggesting that vulnerability to preterm delivery in IUGR pregnancies is induced 206 considerably earlier. 
208 We contend that maintaining a relatively advanced fetus in late pregnant guinea pigs, as in

209 human pregnancy, may increase susceptibility to preterm upregulation of labor-associated

210 pathways and that this susceptibility is further increased following IUGR. We have previously

211 established that PTGS1 rises dramatically with labor in the guinea pig in a similar manner to the

212 increase in expression of the PTGS2 isoform in human labour which is responsible for the

213 dramatic increase in prostaglandin production ${ }^{9}$. The present finding of elevated myometrial

214 PTGS1 expression in IUGR animals suggests that the uterus is exposed to elevated stimulation

215 by prostaglandins for a considerable period prior to labor onset. This supports our contention

216 that IUGR-associated preterm labor is mediated at least in part by increased prostaglandin

217 availability at the uterus. Interestingly while PTGS1 expression rose in amnion and placenta at

218 term, expression was not affected by IUGR. This may suggest that myometrial expression is

219 under the influence of factors that are up-regulated by stress but do not affect PTGS1 in the fetal

220 tissues. In contrast, IUGR had marked effects on HPGD expression in the chorion, possibly

221 indicating different, and as yet unclear IUGR-induced processes controlling the expression of

222 each enzyme.

223

224 HPGD synthesis in the chorion has a key role in maintaining uterine quiescence during 225 pregnancy by providing a protective barrier between the amnion, the main site of prostaglandin 226 synthesis and the myometrium, the site of contractile activity. Previous studies have reported 227 decreases in HPGD expression in the chorion of women and baboons prior to labor onset ${ }^{19,25}$. 228 Consistent with these findings, we observed a marked reduction in HPGD protein expression 229 after labor onset in the sham operated guinea pigs suggesting a loss in expression is needed for 230 normal labor in this species. The observation that HPGD expression in IUGR pregnancy was 
231 reduced from 62 days of gestation compared to control levels supports the contention that IUGR

232 causes changes that are permissive in nature and lead to a pregnancy that is poorly protected

233 from challenges that may raise intrauterine prostaglandin synthesis. The reduction in expression

234 was observed at least a week prior to delivery in the IUGR group of animals suggesting, while

235 these animals did indeed deliver before the controls, they were primed for delivery at an even

236 earlier date. The finding that there was no further fall in HPGD expression with labor onset in

237 IUGR pregnancies again suggests levels of expression were already at sufficiently low levels to

238 permit labor onset well before labor was triggered.

240 The observation that labor occurred in the IUGR animals without a marked rise in amniotic fluid

241 or circulating maternal cortisol suggests that an increase in cortisol immediately prior to labor

242 onset is not required in IUGR pregnancies. The reduced levels of HPGD in these animals may

243 have resulted in a more sensitive environment, enabling labor mechanisms to be initiated without

244 marked changes in circulating cortisol. Unlike species such as the sheep, the fetal HPA axis does

245 not have a central role in the timing of parturition in women and guinea pigs ${ }^{11,26}$ however,

246 increases in cortisol promote fetal maturation, occur in association with labor and likely

247 coordinate aspects of the labor process. Glucocorticoids regulate gene expression in a highly

248 tissue, cell and timing specific manner. For example, glucocorticoids downregulate PTGS2

249 expression in human amnion tissue explants but upregulate PTGS2 in human fetal membranes ${ }^{27-}$

250 . 30 Glucocorticoids, acting via GR, have also been found to down regulate HPGD expression in

251 the chorion ${ }^{31,32}$. The absence of increased cortisol, paired with no change in HPGD expression

252 at labor suggests that IUGR pregnancies were already primed for labor without a further change

253 in HPGD level. Progesterone, reportedly via both PR and GR dependent mechanisms, promotes 
254 uterine quiescence by acting to reduce PTGS and promote HPGD expression ${ }^{31}$. Circulating 255 progesterone concentrations were not found to differ between the control and IUGR pregnancies

256 and therefore unlikely to be responsible for the enzyme changes observed. We have previously

257 found that myometrial PR protein expression decreases prior to labor in the guinea pig ${ }^{10}$, a 258 potential progesterone withdrawal mechanism as circulating progesterone itself does not 259 decrease at labor. Interestingly, we also found myometrial PR expression was maintained at later 260 gestational age in IUGR pregnancies than in sham operated control pregnancies. This may reflect 261 a protective mechanism in the face of increasing PTGS and decreasing HPGD protein expression 262 to maintain uterine quiescence in these compromised pregnancies. It further suggests 263 mechanisms controlling labor differ in IUGR pregnancies.

265 In summary, this study has identified a prostaglandin-driven potential mechanism underlying the 266 association of IUGR with preterm labor. Investigation of other labor-associated proteins such as 267 oxytocin, oxytocin receptor, connexin 43 and the $\mathrm{PGF}_{2 \alpha}$ receptor may provide further insight. 268 Knowledge of upstream induction processes and interventions to inhibit them would be of 269 particular therapeutic advantage given the poor outcome associated with IUGR. We conclude 270 that pregnancies compromised by fetal IUGR demonstrated a reduction in the protective PG 271 metabolism barrier in the chorion likely increasing the vulnerability of these pregnancies to 272 further stimulation leading to preterm labor. Intriguingly, preterm labor in our IUGR model, but 273 not in sham operated controls, has been found to occur without either a reduction in HPGD 274 expression or increase in cortisol concentrations. Further exploration and identification of 275 mechanisms specific to preterm birth in compromised pregnancies may provide targets for 276 preterm labor prevention. 


\section{$278 \quad$ References}

279 1. Morken N-H, Kallen K, Jacobsson B. Fetal growth and onset of delivery: A nationwide 280 population-based study of preterm infants. Am. J. Obset. Gynecol. 2006; 195: 154-161.

281 2. Bukowski R, Gahn D, Denning J, Saade G. Impairment of growth in fetuses destined to 282 deliver preterm. Am. J. Obset. Gynecol. 2001; 185: 463-467.

283 3. Zeitlin J, Ancel PY, Saurel-Cubizolles MJ, Papiernik E. The relationship between 284 Gynaecology. 2000; 107: 750-758.

287 4. Rocha CO, Bittar RE, Zugaib M. Neonatal Outcomes of Late-Preterm Birth Associated or Not with Intrauterine Growth Restriction. Obstetrics and Gynecology International.

5. Garite TJ, Clark R, Thorp JA. Intrauterine growth restriction increases morbidity and mortality among premature neonates. Am. J. Obstet. Gynecol. 2004; 191: 481-487.

292 6. Chwalisz K, Fahrenholz F, Hackenberg M, Garfield R, Elger W. The progesterone 293 antagonist onapristone increases the effectiveness of oxytocin to produce delivery without changing the myometrial oxytocin receptor concentrations. Am. J. Obstet.

296 7. Schellenberg JC. The effect of oxytocin receptor blockade on parturition in guinea pigs. Journal of Clinical Investigation. 1995; 95: 13-19.

298 8. Garfield R, Saade G, Buhimschi C, et al. Control and assessment of the uterus and cervix during pregnancy and labour. Human Reproduction Update. 1998; 4: 673-695. 
300

301

302

303

304

305

306

307

308

309

310

9. Welsh T, Mitchell CM, Walters WA, Mesiano S, Zakar T. Prostaglandin H2 synthase-1 and -2 expression in guinea pig gestational tissues during late pregnancy and parturition. Journal of Physiology. 2005; 569: 903-912.

10. Palliser HK, Zakar T, Symonds IM, Hirst JJ. Progesterone Receptor Isoform Expression in the Guinea Pig Myometrium From Normal and Growth Restricted Pregnancies. Reproductive Sciences. 2010; 17: 776-782.

11. Illingworth DV, Challis JRG, Ackland N, et al. Parturition in the guinea pig; plasma levles of steroid hormones, steroid-binding proteins, and oxytocin, and the effect of corticosteroids, prostaglandins and adrenocorticotrophin. J Endocrinol. 1974; 63: 557570.

12. Thomson AJ, Telfer JF, Young A, et al. Leukocytes infiltrate the myometrium during human parturition: further evidence that labour is an inflammatory process. Human Reproduction. 1999; 14: 229-236.

13. Holcberg G, Huleihel M, Sapir O, et al. Increased production of tumor necrosis factor a (TNF-a) by IUGR human placentae. European Journal of Obstetrics Gynecology and Reproductive Biology. 2001; 94: 69-72.

14. Keelan JA, Blumenstein M, Helliwell RJA, et al. Cytokines, prostaglandins and parturition - a review. Placenta. 2003; 24: S33-S46.

15. Mitchell MD, Goodwin V, Mesnage S, Keelan JA. Cytokine-induced coordinate expression of enzymes of prostaglandin biosynthesis and metabolism: 15hydroxyprostaglandin dehydrogenase. Prostaglandins, Leukotrienes and Essential Fatty Acids. 2000; 62: 1-5. 
322 16. Challis JRG, Lye SJ. Parturition. In: Knobile E, Neill JD, eds. The Physiology of

323 Reproduction. Vol 2. 2nd ed. New York: Raven Press; 1994:985-1031.

324 17. Ellwood DA, Mitchell MD, Anderson ABM, Turnbull AC. The in vitro production of prostanoids by the human cervix during pregnancy: preliminary observations. British Journal of Obstetrics and Gynaecology. 1980; 87: 210-214.

18. Giannoulias D, Patel FA, Holloway AC, et al. Differential changes in 15hydroxyprostaglandin dehydrogenase and prostaglandin H synthase (types I and II) in human pregnant myometrium. The Journal of Clinical Endocrinology and Metabolism. 2002; 87: 1345-1352.

19. Sangha RK, Walton JC, Ensor CM, Tai H, Challis JRG. Immunohistochemical localization, messenger ribonucleic acid abundance, and activity of 15hydroxyprostaglandin dehydrogenase in placenta and fetal membranes during term and preterm labor. The Journal of Clinical Endocrinology and Metabolism. 1994; 78: $982-$ 989.

20. Hirst JJ, Teixeira FJ, Zakar T, Olson DM. Prostaglandin H synthase-2 expression increases in human gestational tissues with spontaneous labor onset. Reprod. Fert \& Dev. 1995; 7: 633-637.

21. Van Meir CA, Matthews SG, Keirse MJNC, et al. 15-hydroxyprostaglandin dehydrogenase: implications in preterm labor with and without ascending infection. The Journal of Clinical Endocrinology and Metabolism. 1997; 82: 969-976.

22. Turner AJ, Trudinger BJ. A Modification of the Uterine Artery Restriction Technique in the Guinea Pig Fetus Produces Asymmetrical Ultrasound Growth. Placenta. 2009; 30: 236-240. 
345 23. Kelleher MA, Palliser HK, Walker DW, Hirst JJ. Sex-dependent effect of a low neurosteroid environment and intrauterine growth restriction on fetal guinea pig brain

24. Lin C, Su S, River L. Comparison of associated high-risk factors and perinatal outcome development. J Endocrinol. 2011; 208: 1-9.

25. Wu WX, Ma XH, Smith GCS, et al. Prostaglandin dehydrogenase mRNA in baboon intrauterine tissues in late gestation and spontaneous labor. American Journal of Physiology. 2000; 279: R1082-R1090.

354 26. Jenkin G, Young IR. Mechanisms responsible for parturition; the use of experimental models. Animal Reproduction Science. 2004; 82-83: 567-581.

27. Wang Z, Tai HH. Interleukin-1B and dexamethasone regulate gene expression of prostaglandin $\mathrm{H}$ synthase-2 via the NF-kB pathway in human amnion derived WISH

28. Mitchell C, Johnson R, Bisits A, Hirst J, Zakar T. PTGS2 (Prostaglandin Endoperoxide Synthase-2) Expression in Term Human Amnion in Vivo Involves Rapid mRNA Turnover, Polymerase-II 5, $\mathrm{A} \leq-$ Pausing, and Glucocorticoid Transrepression. Endocrinology. 2011; 152: 2113-2122.

29. Zakar T, Hirst JJ, Mijovic JE, Olson DM. Glucocorticoids Stimulate the Expression of Prostaglandin Endoperoxide H Synthase-2 in Amnion Cells. Endocrinology. 1995; 136: $1610-1619$.

30. Blumenstein M, Hansen WR, DeVal D, Mitchell MD. Differential regulation in human amnion epithelial and fibroblast cells of prostaglandin $\mathrm{E}_{2}$ production and prostaglandin $\mathrm{H}$ 
synthase-2 mRNA expression by dexamethasone but not tumour necrosis factor-alpha. Placenta. 2000; 21: 210-217.

370 31. Patel FA, Clifton VL, Chwalisz K, Challis JRG. Steroid Regulation of Prostaglandin Dehydrogenase Activity and Expression in Human Term Placenta and Chorio-Decidua in

373 32. Patel FA, Funder JW, Challis JRG. Mechanism of cortisol/progesterone antagonism in the regulation of 15-hydroxyprostaglandin dehydrogenase activity and messenger ribonucleic acid levels in human chorion and placental trophoblast cells at term. The Journal of Clinical Endocrinology and Metabolism. 2003; 88: 2922-2933. 


\section{Figure Legends}

380 Figure 1. Western blots demonstrating specificity of PTGS1 (top panel) and HPGD (bottom

381 panel) primary antibodies alone (left panels) and following incubation with blocking peptides

382 (right panels) in amnion, placenta, myometrium and chorion. Amn, amnion; HPGD, 15-

383 hydroxyprostaglandin dehydrogenase; M, marker; Myo, myometrium; Plac, placenta; PTGS1,

384 prostaglandin endoperoxidase type 1.

386 Figure 2. Relative PTGS1 protein expression in placenta (A) and amnion (B) over late gestation 387 (GA62, 65 and 68) and after labor onset in sham operated control (open bar) and IUGR (closed 388 bar) pregnancies. Placental and amnion PTGS1 expression increased with advancing gestational 389 age however no difference was identified between sham-operated control and IUGR pregnancies. 390 Lower panel demonstrates representative PTGS1 and beta actin loading control western blots. 391 GA, gestational age; IC, internal control; PTGS1, prostaglandin endoperoxidase type 1.

393 Figure 3. Relative PTGS1 protein expression in myometrium over late gestation (GA62, 65 and 394 68) and at labor (A), 2 days prior to expected delivery (B) and representative PTGS1 and beta 395 actin loading control western blot (C) in sham operated control (open bar) and IUGR (closed bar) 396 pregnancies. PTGS1 protein expression was significantly higher in myometrium from IUGR 397 pregnancies at 68 days of gestation (GA) and 2 days (2d) prior to expected delivery. ${ }^{*} \mathrm{P}<0.05$ 398 between control and IUGR within each gestational age group. IC, internal control; PTGS1, 399 prostaglandin endoperoxidase type 1. 
401 Figure 4. Relative HPGD protein expression in chorion over late gestation (GA62, 65 and 68)

402 and at labor (A), a week prior to expected delivery (B) and representative HPGD and beta actin

403 loading control western blots (C) in sham operated control (open bar) and IUGR (closed bar)

404 pregnancies. HPGD expression did not change over gestation or labor in IUGR pregnancies but

405 fell at labor in control pregnancies. HPGD protein expression was significantly lower at

406 gestational age group (GA) 62, 68 and 7 days (7d) prior to expected delivery in IUGR

407 pregnancies compared to control. ${ }^{*} \mathrm{P}<0.05$ between control and IUGR within each gestational

408 age group. HPGD, 15-hydroxyprostaglandin dehydrogenase; IC, internal control.

409

410 Figure 5. Correlation between fetal body weight (g) and relative HPGD protein expression in the

411 chorion at 68 days of gestation. There was a positive correlation between these parameters $(\mathrm{r}=$

$4120.56, \mathrm{P}=0.015$, Spearman). HPGD, 15-hydroxyprostaglandin dehydrogenase.

413

414 Figure 6. Total cortisol concentrations in amniotic fluid (A) and maternal plasma (B) over late 415 gestation and at labor in sham operated control (open bars) and IUGR pregnancies (closed bars).

416 Amniotic fluid cortisol increased in IUGR and control pregnancies at GA68 and at labor 417 compared to GA62, respectively. At labor, amniotic fluid cortisol concentrations were 418 significantly higher in controls than in IUGR. Maternal circulating cortisol concentrations did 419 not change in mothers with IUGR pregnancies and increased at labor in those with normal 420 pregnancies. $* \mathrm{P}<0.05$ between control and IUGR within each gestational age (GA) group. 


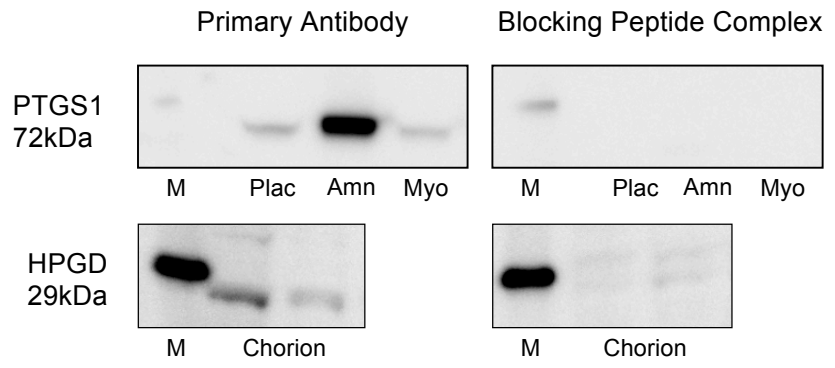

423 Figure 1

A
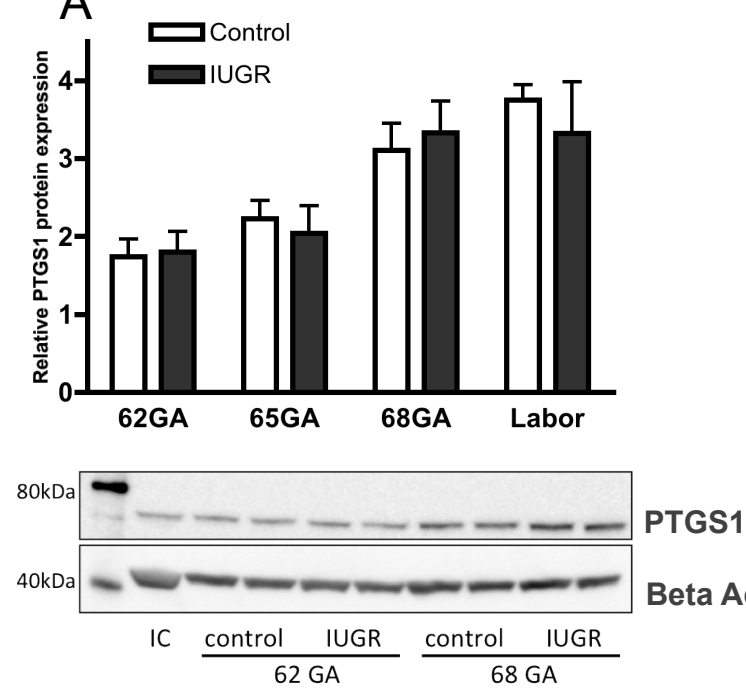

424

425

Figure 2
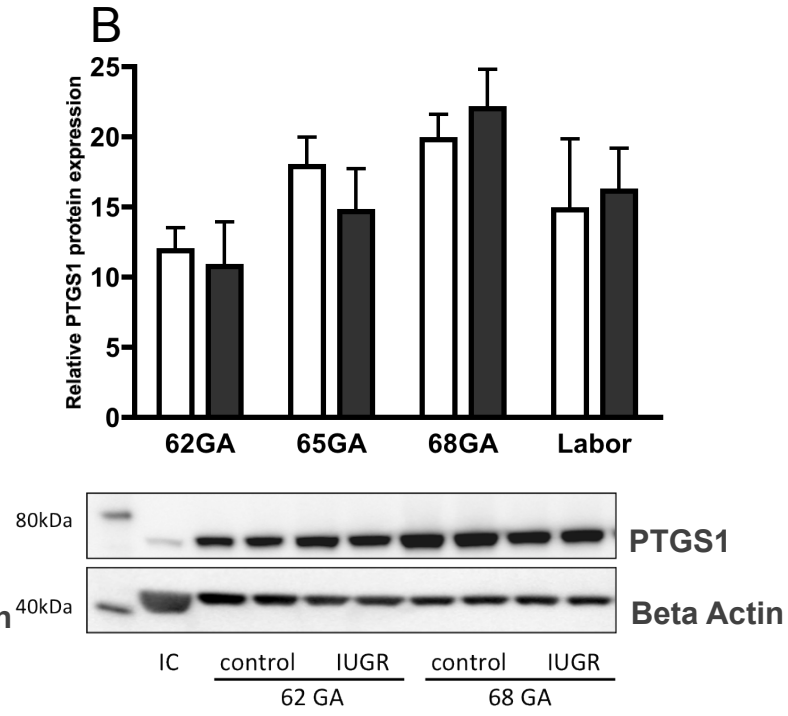


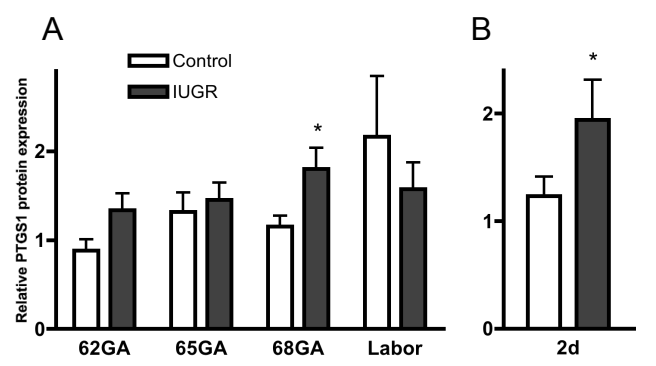

$$
\text { C }
$$

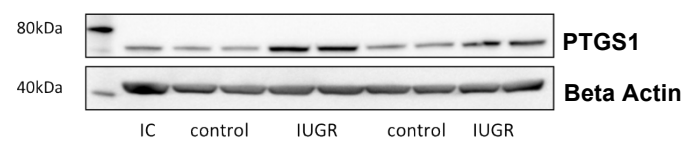

Figure 3

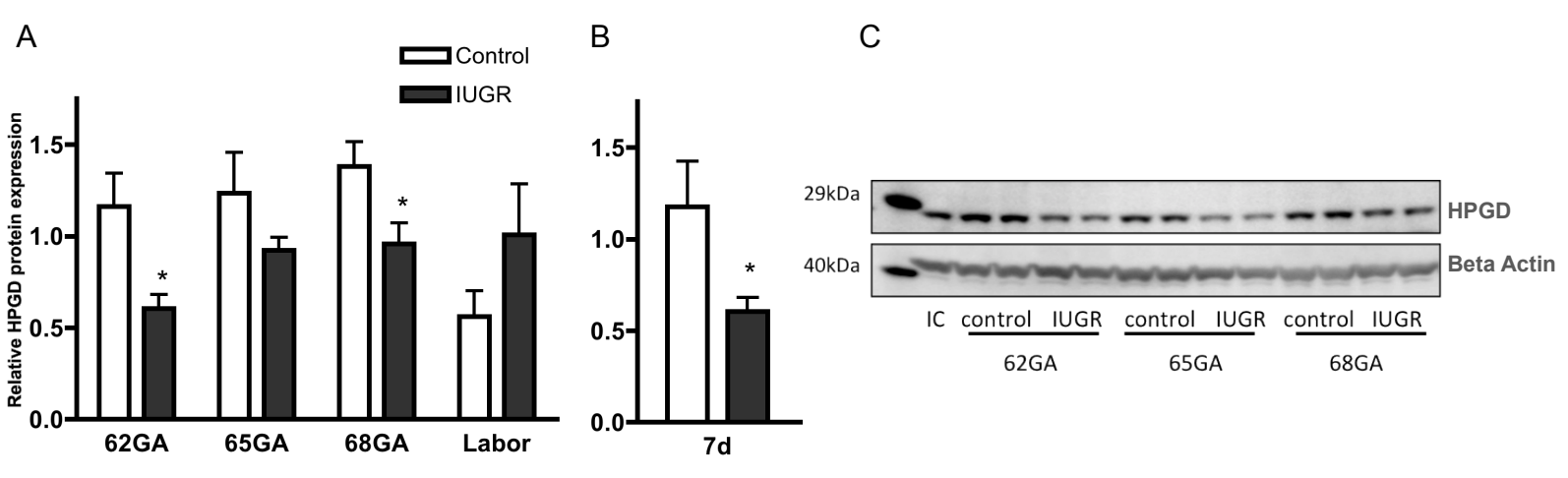

429 figure 4 


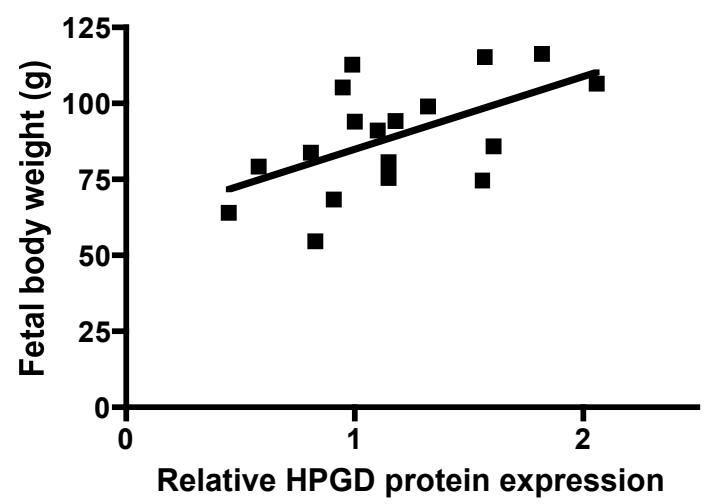

430

431 Figure 5 


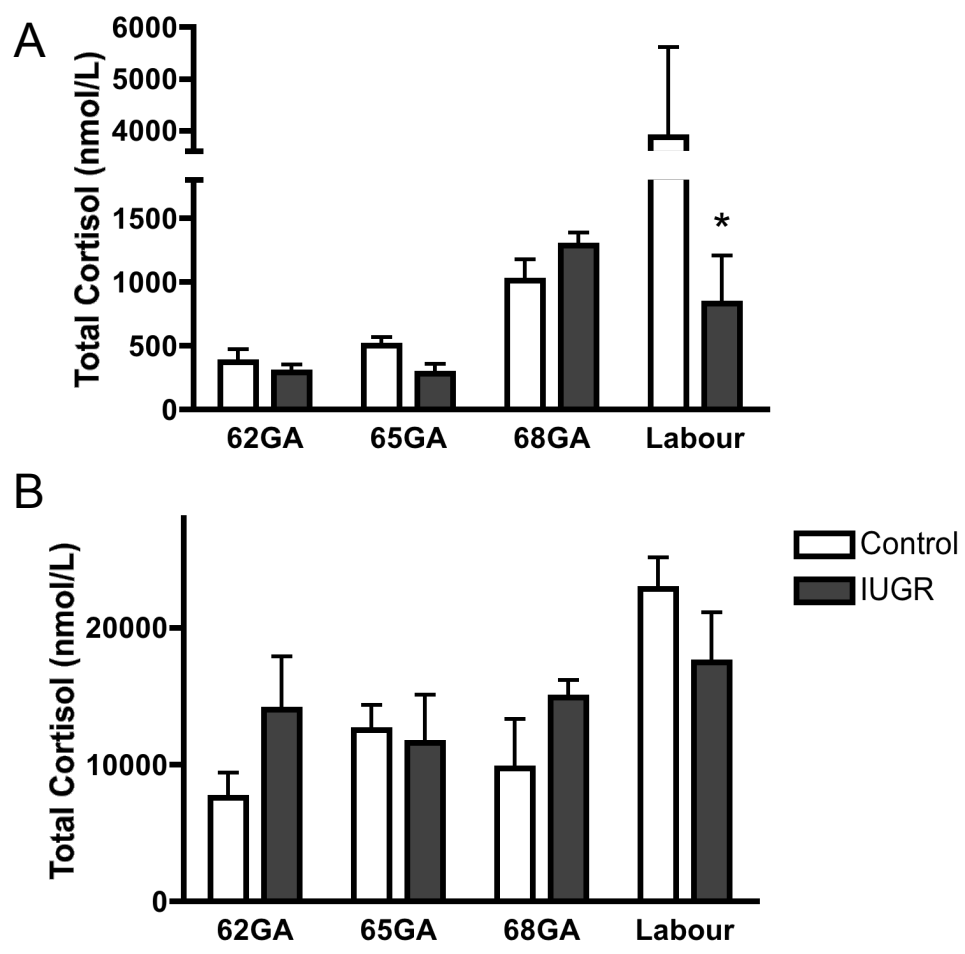

432

433 Figure 6 